\title{
Simulation of aeration of buildings erected on complex terrain
}

\author{
Adham Giyasov, and Dmitry Kim* \\ Moscow State University of Civil Engineering, Yaroslavskoe shosse, 26, Moscow, 129337, Russia
}

\begin{abstract}
The problem of improving the environment by rational use of natural resources is currently very relevant. By means of theoretical and experimental metrological and aerodynamic studies, the infiltration processes between the internal and external air environment during the wind flow around buildings of buildings erected on complex terrain, as well as the adjacent territories of residential development, are established, which allows us to assess the heat loss and ventilation conditions of buildings under wind pressure. A model of the formation of the circulation zone for different geometric parameters of buildings, wind flow velocity and slope steepness is developed, which allows preliminary forecasting of the aeration regime of the adjacent territories. Zones of backwater and calm wind in the adjacent territories were identified. A model of the wind shadow zone has been compiled, which allows the designer to zone the territory adjacent to the building at the design stage, taking into account the aeration regime of the development.
\end{abstract}

\section{Introduction}

In the fight for the purity of the atmosphere and the reduction of harmful emissions into it, natural wind energy is used, which allows aeration of residential areas.

Based on the results of long-term measurements of wind speed and direction carried out at meteorological stations, the wind regime in this area is estimated and graphically depicted in the form of a wind rose diagram.

The problems of improving the air environment in the premises and on the territory, the construction of detached high-rise buildings and their complexes require, in particular, knowledge of the impact of wind on them.

To study the aeration of the building area, it is necessary to find out the distribution of wind speed in the surface area of the building under consideration, as well as to take into account the effect of wind on buildings, which contributes to air filtration through building structures and architectural means that provide air exchange between the internal and external air environment. In this case, an important condition is the assessment of the increase in heat loss of buildings. In addition, the building itself has a significant impact on the nature of the air flow, near the building changes the speed and direction of the wind, there are strong vortex formations around the buildings, depending on the shape in the plan and the spatial solution, as well as on the territory of the building with different planning structure.

The nature of the distribution of wind speed depends on the geometric shape of buildings, on their relative location, so one of the main tasks of aeration is to establish a connection between the geometry of the building and the pressure spectrum on its surface at wind speed and its direction, corrected in the adjacent territories due to the density and planning structure of the building.

\footnotetext{
*Corresponding author: udjinley@gmail.com
} 
For free-standing and unprotected buildings on the plain, methods for calculating the aerodynamic characteristics as a function of the geometric shape of the building have been developed $[1,2,3,4]$.

In the case of a protected building, the aerodynamic performance largely depends on both the shape of the building and the distance between adjacent buildings, their height and relative position.

The area of deceleration of the air flow at the building, associated with eddy formations in its wind-swept area, extends over a distance of about six heights of this building and depends on its shape, parameters and the condition of the wind flow around the building. Outside of this area, the influence of the building on the air flow does not stop, the flow deceleration can be noticeable at a distance of 15 or more of its heights $[5,6,7]$.

\section{Methods}

In residential neighborhoods, natural wind energy is used to reduce air pollution, which improves the microclimate of buildings and the surrounding area, and removes various impurities from the city $[8,9,10]$. When studying the aerodynamics of buildings, you should know the vector direction of the wind, the laminar and turbulent flow of the air flow in the surface area of the building territory, as well as the qualitative and quantitative picture of the wind flow around buildings and their complexes. This process is accompanied by the appearance of a complex vortex zone behind the building and the formation of a wind flow with high turbulence and large deformations of the velocity fields around it and above the building. The influence of these phenomena on the distribution of concentrations of harmful substances between buildings is very significant.

In order to study the formation of circulation zones behind the building, that is, wind shadow zones and the distribution of velocities in them, depending on the geometric dimensions of the buildings, studies were conducted on models of residential buildings in complex terrain, having the shape of a rectangular parallelepiped, the dimensions of which are shown in Table 1, which revealed a number of aerodynamic characteristics around the building.

Table 1. The experimental samples of buildings.

\begin{tabular}{|c|c|c|c|c|c|}
\hline Model No. & $\begin{array}{c}\text { Length } \\
S, m m\end{array}$ & $\begin{array}{c}\text { Width } \\
B, m m\end{array}$ & $\begin{array}{c}\text { Height } \\
H, m m\end{array}$ & $\begin{array}{c}\text { Dimensionless } \\
\text { geometric criteria } \\
Z=\frac{B}{\sqrt{S H}}\end{array}$ & $\begin{array}{c}\text { Length of the } \\
\text { circulation area } \\
\text { behind the } \\
\text { building } \\
X_{\mathrm{C}}\end{array}$ \\
\hline 1 & 400 & 100 & 300 & & $\frac{1}{H}$ \\
\hline 2 & 400 & 100 & 200 & 0,29 & 3,83 \\
\hline 3 & 400 & 100 & 100 & 0,50 & 4,00 \\
\hline 4 & 300 & 100 & 100 & 0,60 & 3,75 \\
\hline 5 & 400 & 200 & 100 & 1,00 & 2,00 \\
\hline 6 & 400 & 400 & 100 & 2,00 & 2,75 \\
\hline
\end{tabular}

The models were tested at the speed of the incoming air flow $v=5 \ldots 20 \mathrm{~m} / \mathrm{s}$ in the working part of the wind tunnel. At the same time, the Reynolds number, depending on the maximum dimensions of the models, was in the range $(1,33 \ldots 4,14) \cdot 10^{6}$. The flow patterns of the models were determined visually by means of silkworms pasted on the black-colored sub-bases of 
the models under study, by smoke-ing the territory from the underground slope of the terrain and recording changes in the wind flow with a video camera. The quantitative assessment of the aerodynamic characteristics of the building was studied using Pitot tubes installed on the characteristic points of the surfaces of the walls of the facades of buildings.

\section{Results}

Summing up the results of experimental aerodynamic studies, the following conclusions are formulated:

When the wind interacts with the building, a backwater zone is formed in front of it and a circulation zone is formed behind it, in the center of which the wind speed decreases to zero.

In the circulation zone, the distribution of air flow velocities along the height is characterized by alternating signs from the zero point up and down, the part of the air flow below the zero-speed line has the opposite direction to the guiding wind flow.

The study of the qualitative picture of the movement of air flows that occurs when the wind flow flows around models of detached buildings, it is established that the flow failure from the windward side of the edge of the cornice of the building in the circulation zone ends both on the inclined surface of the relief behind the windward wall at $<2,5 \mathrm{H}$, and on the roof at $<2,5 H$.

At $<2,5 \mathrm{H}$, two characteristic separation areas are observed: the zone of support in front of the building and the zone of the aerodynamic footprint behind the building (the circulation zone), the length of which depends on the geometric dimensions of the building. In front of the building in the backwater zone, most of the air jet is deflected up and at a point shape in the building plan to the sides and part is lowered down in the opposite direction of the incoming wind flow, forming a mini circulation zone in the windward side of the building.

At a pressure of $\geq 2,5 \mathrm{H}$, the backwater zone and the circulation zones behind the building and at the roof level can be distinguished. The flow disruption with the formation of vortex zones also occurs from the vertical edges of the walls, and end vortex zones arise.

Processing of the results of experimental aerodynamic data of studies on models in a wind tunnel allowed us to establish the dependence of the length of the circulation zone formed behind the building on the length to height of the building $(\mathrm{S} / \mathrm{H})$ of the various windward slope (Fig. 1) and is presented in the form of a calculation formula that takes into account the overall parameters of the building:

$$
X_{C}=K_{C} H\left(\frac{S}{B}\right)^{1 / 2}
$$

where $K_{C}$ is the circulation coefficient, taken according to Table 2 .

The results of aerodynamic model and experimental studies of buildings allowed us to form a model of the circulation zone in the perpendicular direction of the wind, depending on the speed of the incoming air flow, the parameters of the building, the steepness of the slope of the location of the building and is presented in graphical form as a dependence in Fig.1. 
Table 2. The length of the circulation area $(H c)$ and the circulation coefficient $(K c)$.

\begin{tabular}{|c|c|c|c|c|c|}
\hline \multirow{2}{*}{$\begin{array}{c}\text { Slope } \\
\text { steepness, deg }\end{array}$} & $\begin{array}{c}\text { Wind speed } \\
v, \\
m / s\end{array}$ & \multicolumn{4}{|c|}{ Building length, $m / K_{C}$} \\
\cline { 3 - 6 } & & $60 / K_{\mathrm{C}}$ & $80 / K_{\mathrm{C}}$ & $100 / K_{\mathrm{C}}$ & $120 / K_{\mathrm{C}}$ \\
\cline { 3 - 6 } & 2 & $4,6 \mathrm{H} / 2,06$ & $4,8 \mathrm{H} / 1,86$ & $5,0 \mathrm{H} / 1,74$ & $5,2 \mathrm{H} / 1,65$ \\
& 4 & $5,5 \mathrm{H} / 2,47$ & $5,7 \mathrm{H} / 2,21$ & $5,9 \mathrm{H} / 2,05$ & $6,1 \mathrm{H} / 1,93$ \\
& 6 & $6,8 \mathrm{H} / 3,05$ & $6,9 \mathrm{H} / 2,67$ & $7,1 \mathrm{H} / 2,47$ & $7,3 \mathrm{H} / 2,31$ \\
\hline 30 & 2 & $3,5 \mathrm{H} / 1,57$ & $3,6 \mathrm{H} / 1,4$ & $3,8 \mathrm{H} / 1,32$ & $4,0 \mathrm{H} / 1,27$ \\
& 4 & $4,2 \mathrm{H} / 1,88$ & $4,3 \mathrm{H} / 1,67$ & $4,5 \mathrm{H} / 1,56$ & $4,6 \mathrm{H} / 1,46$ \\
& 6 & $5,3 \mathrm{H} / 2,38$ & $5,1 \mathrm{H} / 2,0$ & $5,5 \mathrm{H} / 1,91$ & $5,6 \mathrm{H} / 1,44$ \\
\hline
\end{tabular}
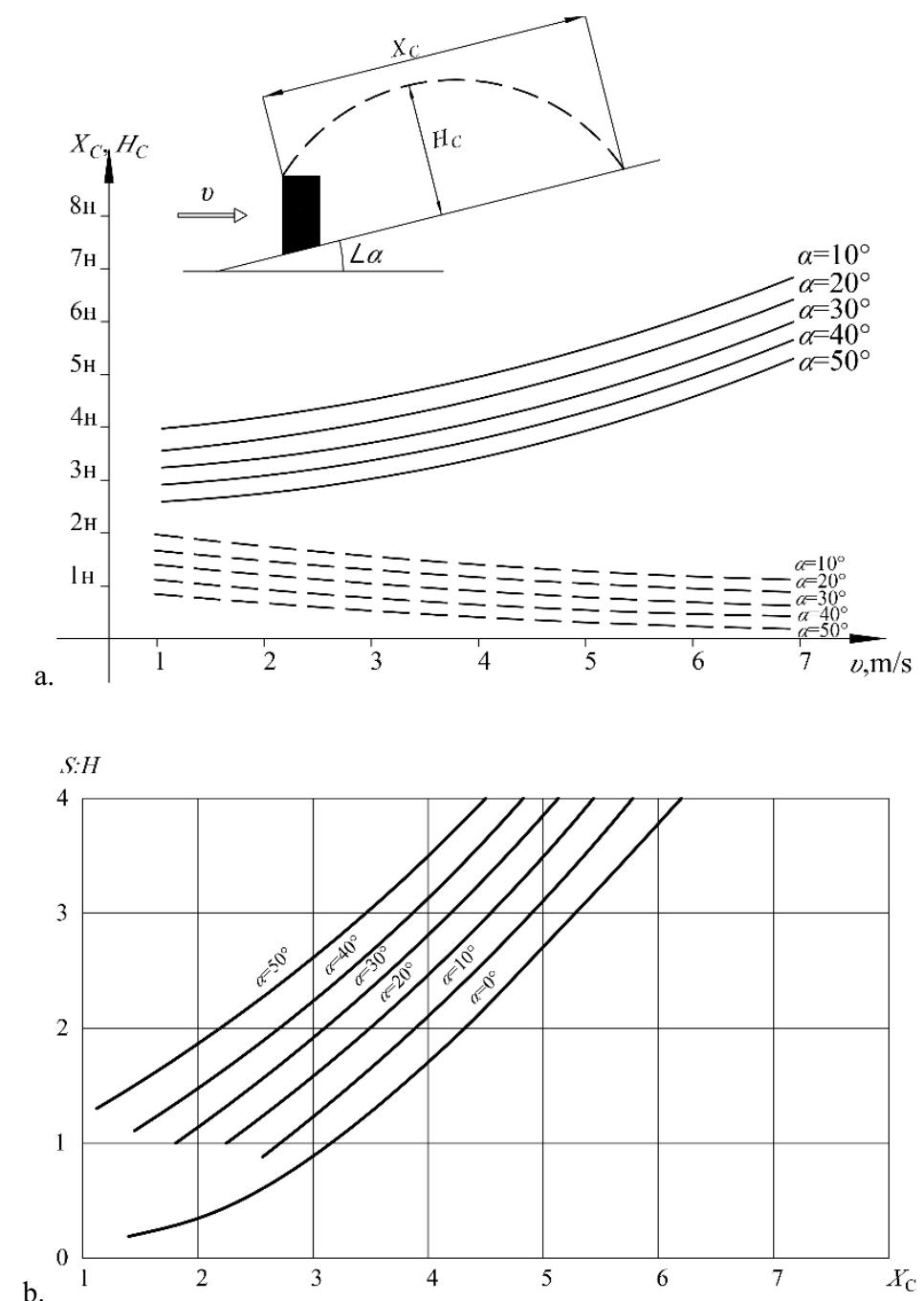

Fig. 1. Model of the formation of the circulation zone in the perpendicular wind direction: $\mathrm{a}$ - at different wind speeds and slope steepness, b - at slope steepness $\alpha$, the ratio of the length and height of the building. 
When a building is located as part of a development, the assessment of its degree of protection during aeration depends on the planning decision and the direction of the wind flow, which determines the area of wind calm, or the increase in the speed of the wind flow forming drafts.

In residential buildings with a large number of buildings located at different angles to the direction of the wind flow, the speed spectrum becomes so complex that it becomes difficult to take into account the security of each building. Therefore, when solving aeration problems, models of entire fragments of urban development are tested in a wind tunnel, which is a timeconsuming test in terms of quantitative assessment. In this regard, at present, to solve such complex problems in the qualitative and quantitative assessment of the aeration of complex shapes in terms of volume and spatial solutions of morphological construction, a software package is used Autodesk CFD, which determines the directions of our further research.

Studies conducted on models of high-rise buildings in wind tunnels in order to identify the inflow and outflow of air mass through oppositely located openings (vents or transoms) of premises in relation to the direction of the slope of the terrain and the incoming air flow allowed us to determine the amount of flowing and flowing air per unit of time in the openings from the windward and windward sides of the building facades (Fig. 2).

The conducted studies of the wind tunnel in order to assess the aeration of buildings and premises of high-rise buildings and the processing of the results allowed us to identify the amount of air inflow and outflow through the supply and exhaust openings or open vents or transoms of light transmission located in opposite facades with through ventilation. (Fig. 2):

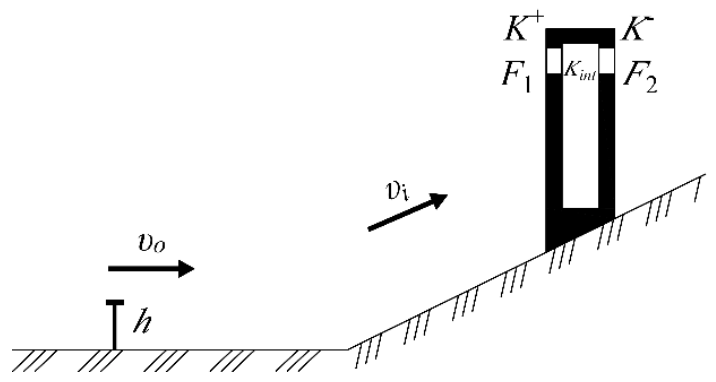

Fig. 2. Layout of air inflow and outflow openings in the building.

For the air supply of the winded facade:

$$
G_{1}=\mu_{1} \cdot v_{0} \cdot \gamma \cdot F_{1} \cdot \sqrt{\left(K_{1}-K_{i n t}\right)}
$$

For the air outflow of the windward facade:

$$
G_{2}=\mu_{2} \cdot v_{0} \cdot \gamma \cdot F_{2} \cdot \sqrt{\left(K_{\text {int }}-K_{2}\right)}
$$

where $G_{1}, G_{2}$ - the amount of air flowing per unit of time through holes 1 and $2 ; \mu_{1}, \mu_{2}$ - the flow coefficients; $v_{0}$ - the air flow velocity (according to the meteorological station), $\mathrm{m} / \mathrm{s} ; \gamma$ - the specific weight of air, $\mathrm{kg} / \mathrm{m}^{3} ; F_{1}, F_{2}$ - - the area of holes 1 and 2 in $\mathrm{m}^{2} ; K_{1}, K_{2}$ - the aerodynamic coefficients for holes 1 of the windward and 2 of the windward side of the facade wall; $K_{\text {int }}$ - the internal aerodynamic coefficient in the presence of two holes, is determined according to [1] by the formula:

$$
K_{\text {int }}=\frac{K_{1} F_{1}^{2}+K_{2} F_{2}^{2}}{F_{1}^{2}+F_{2}^{2}}
$$


The value of the aerodynamic coefficients in the norms is given for windward $(+0,8)$ and windward $(-0,4)$ buildings erected on the plain. These coefficients for complex terrain largely depend on the steepness of the slope, the geometric dimensions and shape of buildings and their relative positions $[18,19,20]$.

The study of changes in the air flow velocity along the hill slope allowed us to establish the following dependence:

$$
v_{i}=v_{0} \exp \sqrt{21,16\left(1-\bar{x}^{2}\right)} \frac{\bar{\delta}}{\pi}
$$

where $\bar{x}=\frac{x}{L}-$ relative coordinate; $\bar{\delta}=\frac{\delta}{L}-$ relative height; $L-1 / 2$ width of the hill, $\mathrm{m} ; v_{i}-$ the speed of the air flow over the considered part of the hill, $\mathrm{m} / \mathrm{s} ; \delta-$ the height of the hill elevation, $\mathrm{m} ; x$ - the distance from the top of the hill of the considered building, $\mathrm{m}$.

Substituting formula 4 in formulas 2 and 3, the following calculation formulas are obtained, which determine the amount of air flowing per unit time through the holes 1 and 2 of the building under study on the slope of the terrain:

$$
\begin{aligned}
& G_{1}=\mu_{1} \cdot v_{0} \cdot \exp \sqrt{21,16\left(1-\bar{x}^{2}\right)} \frac{\bar{\delta}}{\pi} \cdot \gamma \cdot F_{1} \cdot \sqrt{\left(K_{1}-K_{\text {int }}\right)} \\
& G_{2}=\mu_{2} \cdot v_{0} \cdot \exp \sqrt{21,16\left(1-\bar{x}^{2}\right)} \frac{\bar{\delta}}{\pi} \cdot \gamma \cdot F_{2} \cdot \sqrt{\left(K_{\text {int }}-K_{2}\right)}
\end{aligned}
$$

The calculated formulas for the intensity of the inflow and outflow of air exchange through the opening reflect the air exchange between the internal and external environment of buildings, depending on the location of the slope height and can be used to assess the aeration of the premises of the designed buildings and the multiplicity of air exchange of premises, determined further by the formula:

$$
v_{p}=\frac{G_{2}}{V_{p} \cdot \gamma_{a}}
$$

where $v_{p}$ - is the volume of the room, $\mathrm{m}^{3} ; \gamma_{a}$ - is the air density, $\mathrm{kg} / \mathrm{m}^{3}$.

When the direction of the air flow is perpendicular to the long facade of a rectangular building, the value of the aerodynamic coefficients, depending on the geometric parameters

\begin{tabular}{|c|c|c|}
\hline \multirow{2}{*}{$\begin{array}{l}\text { Size ratio calculation } \\
\text { formula }\end{array}$} & \multicolumn{2}{|c|}{ Building wall facing the wind } \\
\hline & Longitudinal & Transverse \\
\hline Wall aspect ratio & windward at $0,18 \leq Z \geq 1,1$ & windy at $0,18 \leq Z \geq 1,1$ \\
\hline Calculation formulas & $K_{90}^{+}=\frac{0,73}{Z_{90}^{+}}+0,42$ & $K_{90}^{-}=\frac{0,045}{Z_{90}^{-}}+0,344$ \\
\hline
\end{tabular}
of the buildings, can be determined using the tracking formulas in Table 3.

Table 3. Formulas for calculating the aerodynamic coefficients for detached buildings with a perpendicular wind flow. 
where $Z=\frac{\bar{B}}{\bar{S}^{n}}, \bar{B}=\frac{B}{H}, \bar{S}=\frac{S}{H}, n=\frac{1}{4}, B, H, S-$ are the width, height, and length of the building, respectively, $\mathrm{m} ; Z-$ is a dimensionless geometric simplex; $\bar{B}, \bar{S}-$ is the relative width and height of the building.

In the direction of the wind flow to the main facade of the building on the side walls of the facades of the building perpendicularly, the suction effects of the wind flow are observed, while the aerodynamic coefficients on the wall surfaces range from $-0,34$ to- $-0,64$.

\section{Discussion of results}

As a result of the conducted research in a wind tunnel on models of buildings with different parameters and shapes, the following indicators of excessive wind pressure on the walls of building facades are important for designers, which can be the initial data in the design of enclosing parts of multi-storey and high-rise buildings erected in conditions of difficult terrain. Particularly, it is noted that in the longitudinal walls of the facades of all the buildings under study, the aerodynamic coefficient is in the range from $+0,52$ to $+0,83$, which causes excessive wind pressure in the center of the plane of the walls of the facade of buildings from $+0,66$ to $+0,68$, with the removal to the outskirts of the walls of the facade of buildings in the direction of the end facades, the aerodynamic coefficient falls to $+0,36 \ldots+0,38$ by 0,3 to 0,4 building heights. With the approaching the cornice part of the facade of buildings, the aerodynamic coefficients are reduced to $+0,54 \ldots+0,58$.

\section{Conclusions}

Summing up the results of the study in general, it is noted that the present method of calculating and predicting the aeration regime of buildings constructed on complex terrain in the form of air inflow and outflow at wind pressure, as well as qualitative and quantitative aerodynamic characteristics of the adjacent territories, allows us to solve a number of problems in the field of improving the energy efficiency of buildings by identifying wind loads on buildings and infiltration heat losses.

\section{References}

1. E.I. Retter, Architectural and construction aerodynamics, Stroyizdat, 294, (1984)

2. F.L. Serebrovsky, Aeration of populated areas, Stroyizdat, 170, (1985)

3. O.I. Poddaeva, I.V. Dunichkin, Architectural and construction aerodynamics, Vestnik MGSU, 6, 602-609 (2017)

4. I.K. Lifanov, V.A. Gutnikov and other, Modeling of aeration in the city, Dialogue, 134 (1994)

5. K.I. Semashko, Guidelines for assessing and regulating the wind regime of residential buildings, Stroyizdat, 64 (1986)

6. A. Giyasov, Modeling of aeration of buildings and structures erected in a mountain valley. International Multi-Conference on Industrial Engineering and Modern technologies IOP Conf. Series: Materials Science and Engineering, 463 (2018)

7. A.I. Giyasov, B.I. Giyasov, Sh.R. Gamzaev, Residential development in difficult terrain and hot climate, Housing construction, 3, 26-27 (2006) 
8. A. Giyasov, O.N. Sokolskaya, Formation of urban development taking into account the environmental factors of the atmospheric environment in hot, low wind and calm conditions, Print Terra, 140 (2016)

9. A.A. Rizka, G.P. Henze, Improved airflow around multiple rows of buildings in hot arid climates, Energy and Buildings, 42, 1711-1718 (2010)

10. C. Ghiaus, F. Allard, M. Santamouris, C. Georgakis, F. Nicol, Urban environment influence on natural ventilation potential, Building and Environment, 41, 395-406 (2006)

11. J.M. Herbert, R.D. Herbert, Simulation of the effects of canyon geometry on thermal climate in city canyons, Mathematics and computers in simulation, 59, 243-253 (2002)

12. A.A. Magai, V.S. Zyryanov, E.Yu. Shalygina, The importance of special technical conditions for the design of high-rise buildings, Housing construction, 11, 17-20 (2015)

13. V. Ivanov, A. Ivanova, Study of the aerodynamic regime of the cooling system of the foundations of buildings on the filling soil in the conditions of the far north. In the collection: International scientific conference on energy, environmental and construction engineering, 10005 (2018)

14. H. Golasz-Szolomicka, J. Szolomicki, Architectural and structural analysis of selected twisted tall buildings. In the collection, IOP conference series: Materials science and engineering, 052050 (2019)

15. R.N. Guzeev, A. Domaingo, Long span bridges buffeting response to wind turbulence, Magazine of civil engineering, 1, 35-49 (2020)

16. G. Solari, Wind actions and effects on structures, Springer tracts in civil engineering, 655801 (2019)

17. A.P. Trunev, Theory of turbulence and modeling of turbulent transport in the atmosphere, Polythematic network electronic scientific journal of the Kuban State Agrarian University, 59, 1-25 (2010)

18. E.A. Ilyukhina, S.I. Lakhman, A.B. Miller, V.I. Travush, Structures of the high-rise building "Lakhta center" in Saint-Petersburg, IJCCSE, 15, 14-39 (2019)

19. S. Makhortykh, A. Derguzov, Simulation of wind effects on high-rise buildings, In the collection: 19th international multidisciplinary scientific geoconference sgem conference proceedings, 951-958 (2019)

20. A. Titov, S. Khrapov, V. Radchenko, A. Khoperskov, Aerodynamic models of complicated constructions using parallel smoothed particle hydrodynamics. In the collection: Proceedings of the international conference. Supercomputer consortium of Russian universities, Russian academy of sciences, 31-42 (2018) 